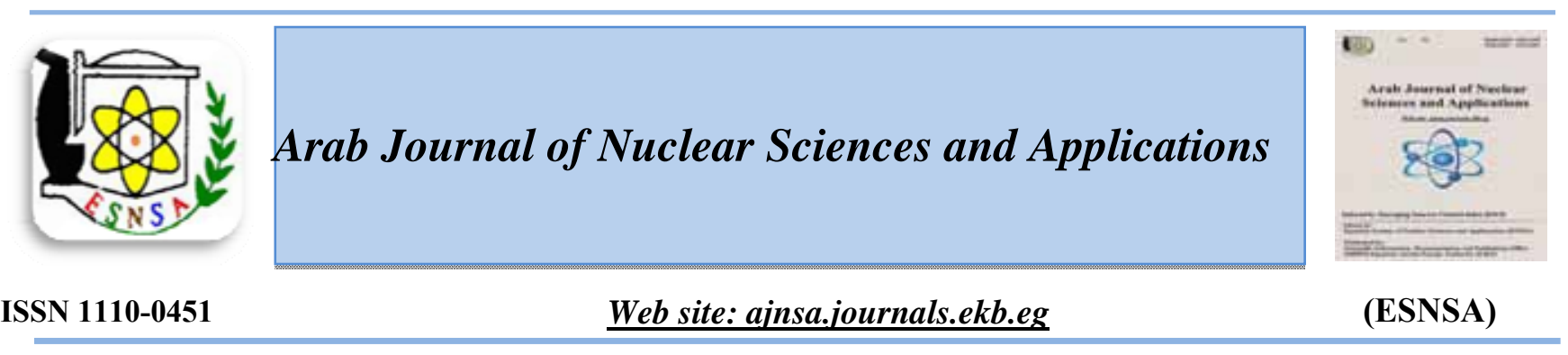

\title{
Role of Loss Mechanisms in the Breakdown of Argon Irradiated with IR Laser Radiation
}

\author{
O. Aied Nassef ${ }^{(1)}$ and Yosr E.E-D Gamal ${ }^{(1)}$ \\ ${ }^{1}$ National Institute of Laser Enhanced Sciences, Cairo University, Giza, Egypt
}

\begin{abstract}
Received $4^{\text {th }}$ June 2017 Threshold intensity measurements of the breakdown of argon are numerically analyzed. The breakdown Accepted $24^{\text {th }}$ Aug.2017 is induced by a Nd: YAG laser radiation at wavelength of $1064 \mathrm{~nm}$ and pulse duration of $8.5 \mathrm{~ns}$.The investigation considered argon over pressure range extended from 25 torr- 760 torr.The measured threshold intensity corresponding to this pressure range is found to vary between $5.4 \times 10^{11} \mathrm{~W} / \mathrm{cm}^{2}$ and $1.1 \times 10^{11} \mathrm{~W} / \mathrm{cm}^{2}$. These are the experimental conditions given by Davis et al. [1]. The analysis considers an electron cascade model given previously by Evans and Gamal (1980) [2] and modified by Gamal and Shafik [3]. The model solves the time dependent energy equation numerically in addition to a set of equations describing the rate of change of the formed excited states population. For realistic results, most of the physical processes that might take place during the interaction of laser radiation with the gas are included in the modified model. The calculated threshold intensity showed reasonable agreement with the measured values over the tested pressure range. The obtained values of the electron energy distribution function as well as the time variation of the electrons density for selected gas pressures, revealed that photo-ionization of the formed excited states plays a minor role for electron generation and plasma formation over the studied gas pressure range. Collisional ionization is the main mechanism responsible for the breakdown process. The loss process, due to electron diffusion, is found to have significant contribution only at pressures $\leq \mathbf{4 0}$ torr. On the other hand, the recombination process is acting in a pronounced manner for the higher pressure regime.
\end{abstract}

Keywords: Laser induced argon plasma, inverse Bremsstrahlung absorption, electronic excitation, electron recombination

\section{Introduction}

The breakdown phenomenon induced by pulsed laser sources is of a great importance in various applications. These include micro industries in electronics that use the plasma etching technique in the integrated circuits, as well as in the environmental application for the measurement of pollution in air, water and soil and surface cleaning. It has also wide applications in medicine and biology. The later applications opened new doors for diagnostics, therapy, and surgery such as eye microsurgery for diabetics. Such applications depend mainly on the plasma formed in the focal volume at the breakdown region. The nature of this plasma is found to vary according to the laser characteristics and the type of the medium.

During the eighties of the last century, it was possible to obtain laser source that operates at different wavelengths covering a range from IR up to VUV of the electromagnetic radiation. This source has been used by different research groups $[1,4-8]$ to carry out measurements on the variation of the threshold intensity with the laser wavelength in the breakdown of atomic and molecular gases. This study is devoted to analyzing the dependence of the threshold intensity on both laser wavelength and gas pressure. All the measurements showed

Corresponding author: onassef@niles.edu.eg

DOI: 10.21608/ajnsa.2018.12717

(C) Scientific Information, Documentation and Publishing Office (SIDPO)-EAEA 
that threshold intensity is peaking at wavelength of $1064 \mathrm{~nm}$, independent of the value of gas pressure or the nature of the gas. No explanation was given for this behavior.

Therefore in this work, a theoretical study is performed to investigate the origin of this peak observed by Davis et al. [1] in the measurements that carried out to study argon breakdown. The breakdown is induced by the fundamental frequency of $\mathrm{Nd}$ : YAG laser radiation at wavelengths $1064 \mathrm{~nm}$ with pulse duration $8.5 \mathrm{~ns}$. The measurements considered argon over a pressure range extending from 25 torr -760 torr. The investigation is based on a modified electron cascade model by Gamal and Shafik [3] previously developed by Evans and Gamal [2]. This model successfully interpreted the experimental measurements that were carried out to study the phenomenon of laser-induced breakdown of $\mathrm{Xe}$ [9], $\mathrm{He}$ [10] and $\operatorname{Kr}$ [11]. The modified model solves the time dependent energy equation numerically for the electron energy distribution function (EEDF), simultaneously with a set of equations that describe the rate of change of the excited atoms population. The formulation takes into account most of the possible physical processes that can occur during the interaction of laser radiation with argon gas. In this model, it is considered that the electron energy gain from the laser field during the Inverse Bremsstrahlung process followed by inelastic collision leads to the excitation and ionization of the gas atoms. In addition, multi-photon ionization of the excited atoms and inelastic collisional ionization processes are the responsible mechanisms for electron generation and ultimately gas breakdown. The model also considered the loss processes that diminish the electrons density or their energy. This gas is deliberately chosen since it showed a minimum Ramseur in the relation between the momentum transfer collisional frequency and the electron energy. This minimum might have a noticeable effect on the rate of gain of the energy of the electrons from the laser field during the inverse Bremsstrahlung absorption process which plays an important role in the breakdown of argon by the used laser wavelength. For realistic results, the computation cared for the exact correlation between the electron energy and cross-section and rate coefficients of each physical process encountered in this model.

\section{The Model Description}

The theoretical approach used to describe the electron cascade model is given in details previously by Evans and Gamal [2] and Gamal et al. [3]. Our calculations are based on an argon atom model that comprises four energy states namely: ground state, two electronics excited states; at $11.6 \mathrm{eV}$ and $13.2 \mathrm{eV}$, as well as the ionized state $15.85 \mathrm{eV}$.

Consequently, the following collisional and radiative processes are considered in the model:

(I). Electron inverse Bremsstrahlung absorption.

(II). Electron impact excitation of the lower state, by electrons of energy $\epsilon>11.6 \mathrm{eV}$.

(III). Electron impact excitation of the upper state, by electrons of energy $\epsilon>13.2 \mathrm{eV}$.

(IV). Electron impact ionization of ground state atoms with electrons having energies $\epsilon>$ $15.85 \mathrm{eV}$.

(V). Collisional ionization from the lower excited state by electrons having energies $\epsilon>15$. $85-11.6 \mathrm{eV}$.

(VI). (vi) Collisional ionization from the upper excited state by electrons having energies $\epsilon>15.85-13.2 \mathrm{eV}$.

(VII). Photo-ionization of the lower excited state at $11.6 \mathrm{eV}$.

(VIII). Photo-ionization of the upper excited state at $13.2 \mathrm{eV}$.

(IX). Recombination losses.

(X). Diffusion of electrons out of the focal volume.

Following to these physical processes, the energy gained from the laser field by electrons is given by the equation:

$$
\frac{\partial n(\varepsilon, t)}{\partial t}=\frac{2 \varepsilon}{3 m v_{m}(\varepsilon)} \nabla^{2} n+\frac{1}{3} \varepsilon_{0} v_{m}(\varepsilon) \frac{\partial n}{\partial \varepsilon}+\frac{2}{3} \varepsilon_{0} v_{m}(\varepsilon) \frac{\partial^{2} n}{\partial \varepsilon^{2}}+I C T
$$


where $\varepsilon_{0}=\mathrm{e}^{2} \mathrm{E}^{2} / 2 \mathrm{~m} \omega^{2}$ is the average oscillatory energy of an electron in the laser field with electric field $\mathrm{E}$ and angular frequency $\omega$, e and $\mathrm{m}$ are the electronic charge and mass. $v_{\mathrm{m}}(\varepsilon)$ is the momentum transfer collision frequency and $\mathrm{n}(\varepsilon)$ is the electronic density at energy range $\varepsilon, \varepsilon+\mathrm{d} \varepsilon$.

The first term on the RHS of the equation represents the electron diffusion out of the focal volume. The second term signifies the energy gained by electrons from the laser field. The third term refers to the energy diffusion along the energy axis. The inelastic collision term, ICT includes the excitation, ionization, and two step ionization processes as well as electron loss through recombination.

\section{Argon data}

The collision cross section of the momentum transfer for argon showed a strong energy dependence which is manifested by the Ramseur minima at 0.3 and $0.4 \mathrm{eV}$. Using curve fitting technique to the data given by Hayatshi (1981) [12], an analytical formula for this cross section is given by Gamal and Azzouz [13].

$$
\begin{gathered}
\sigma_{m}=10^{-15} /\left(1+1446 \epsilon-258331 \epsilon^{2}\right. \\
\left.+35035 \epsilon^{3}-85868 \epsilon^{4}\right) \\
0.0<\varepsilon<0.3 \mathrm{eV}
\end{gathered}
$$

Then, the collision frequency $v_{\mathrm{m}}$ is deduced from the relation:

$$
V_{m}=N \sigma_{m}\left(\frac{2 \varepsilon}{m}\right)
$$

where $\mathrm{N}$ is the gas density per unit volume The various relevant cross sections and rate coefficients of the physical mechanisms responsible for the production and loss of electrons and their energy considered in the present model are adopted from Weyl and Rosen [14] and shown in our previous work Gamal and Shafik. [3].

\section{Method of calculation}

The first and second derivatives shown in equation (1) are solved numerically using a finite difference technique. The linear part of the equation is solved applying step-by-step integration method. In doing so, two lengths of the time step are used in the calculations. The shorter time step is used when an Arab J. Nucl. Sci. \& Applic. Vol. 51, No. 4 (2018) elastic collision takes place for the energy gain and loss terms, while the longer one is considered in the calculations of the inelastic collisional terms. In this work, it is assumed that the interaction starts with one electron present at the focal volume before firing the laser. For continuation of the EEDF, the energy of this electron is assumed to have a Gaussian distribution with its peak energy located at $4 \mathrm{eV}$ (which equals about one fourth of the ionization potential of the gas). The temporal change of the laser intensity is assumed to be Gaussian shape. The breakdown criterion adopted in this work is the attainment of ionization of $0.1 \%$ of the atoms present in the focal volume.

The computer program incorporates the electron energy equation as well as the rate equations for the formed excited atoms in addition to the formulae which represent the rate and rate coefficient of the considered physical processes. The computations revealed the relation between the threshold intensity and gas pressure. To validate the model, the obtained thresholds are then compared with the experimentally measured ones.

\section{Results and Discussion}

Breakdown threshold versus gas pressure

Computations are performed under the experimental conditions of Davies et al. [1]. This experiment is carried out investigate the threshold intensity dependence on the gas pressure for argon breakdown induced by $\mathrm{Nd}$ : YAG laser radiation at wavelengths $1064 \mathrm{~nm}$ with pulse duration $8.5 \mathrm{~ns}$ under a pressure ranging from 25 torr to 760 torr. Figure (1) shows the calculated thresholds (solid curve) as a function of gas pressure. To facilitate the comparison, the experimentally measured ones (scattered solid circles) are also shown in this figure. The results showed a reasonable agreement between the calculated and measured thresholds over the tested pressure range. This agreement validates the model.

Figure 2 illustrates the EEFD calculated at the peak curve (a) and end curve (b) of the laser pulse for the low (Figure2i) and high (Figure2ii) pressure values. It is clear from this figure that the behavior of the EEDF varies with the gas pressure 


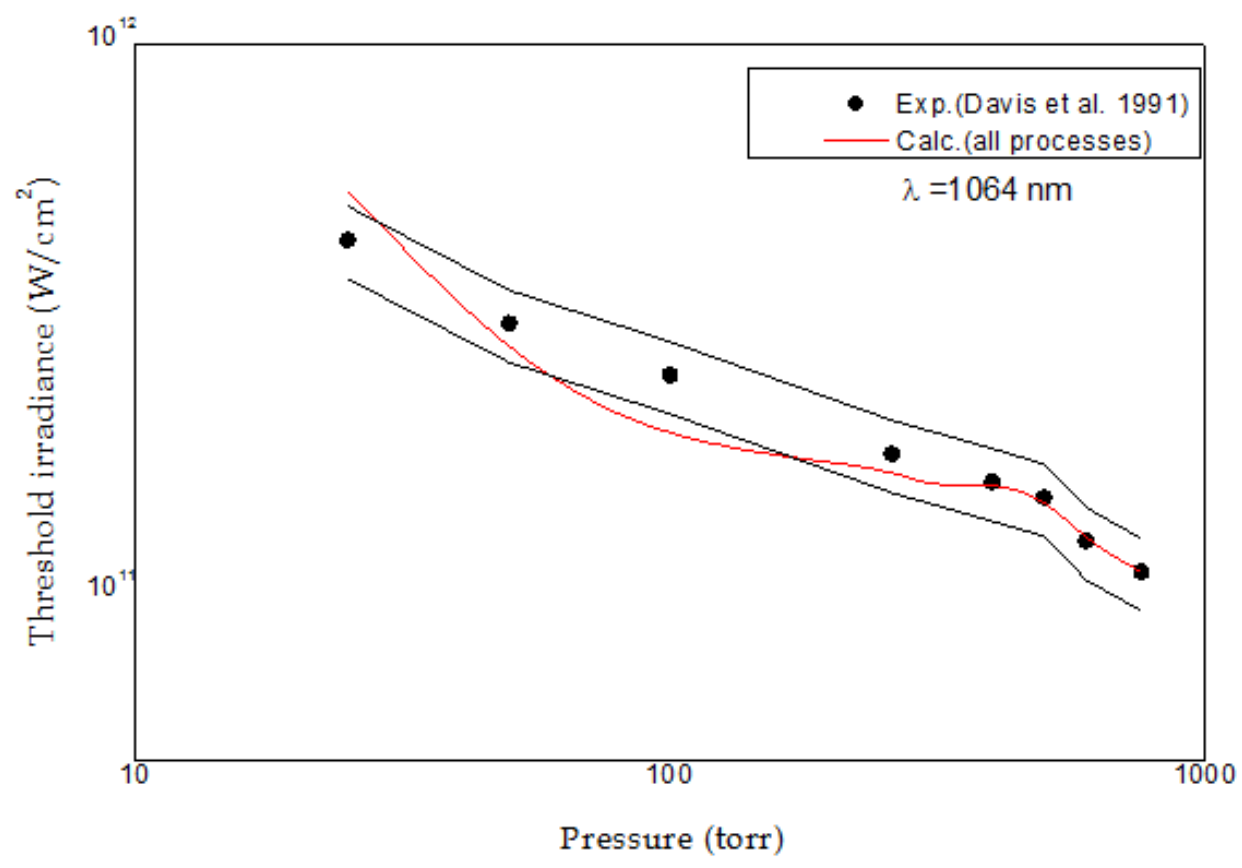

Figure(1): Comparison between the calculated threshold intensity in the presence and absence of electron loss processes and measured values of Davis et al. (1991) as a function of gas pressure when a laser beam of wavelength $1064 \mathrm{~nm}$ induces breakdown in argon

It is found that at the low pressure (Figure2i), the EEDF at the peak (curve a) lies below that obtained at the end (curve b). This result justifies that electron generation via multiphoton absorption and collisional processes exceeds electron loss by diffusion during the descending phase of the laser pulse. At the high pressure, however, the

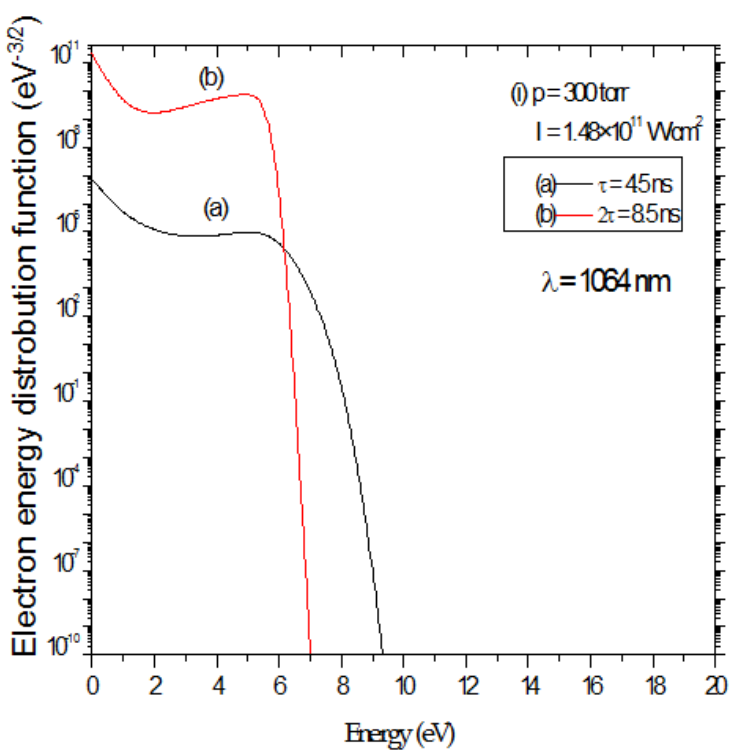

calculated the EEDF proved that ionization leading to gas breakdown depends mainly on collisional processes. This is clear from the close values of the EEDF at both the peak (curve a) and the end (curve b) of the laser pulse shown in Figure(2ii).

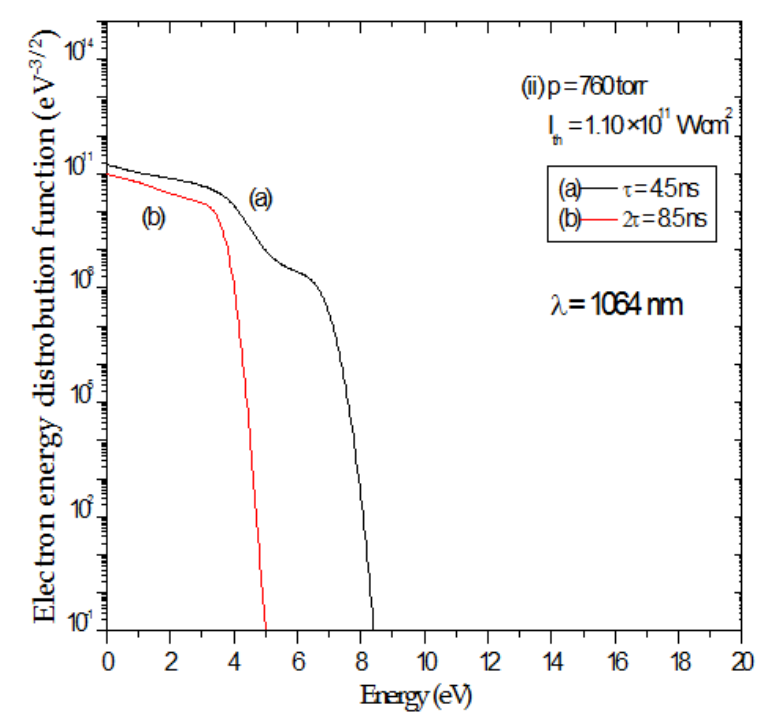

Figure (2): The temporal variation of the calculated EEDF during the laser pulse for gas pressure (1) 300 torr and (2) 760 torr at the peak (curve a) and end (curve b) of the pulse 
To get a deeper insight into the important role played by ionization processes as well as loss processes during the laser pulse, a study is performed to obtain the temporal variation of the electron density at the two pressure values. This is shown in Figure(3).

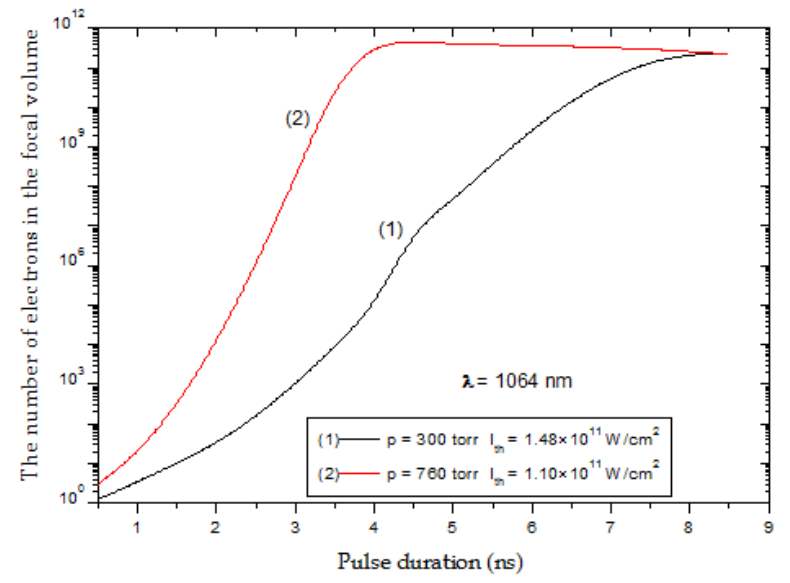

Figure (3): The variation of the number of electrons in the focal volume during the laser pulse calculated at the selected values of gas pressure

This figure demonstrated that at the low-pressure value (300 torr) the electron density exhibited a gradual increase over the whole pulse length as shown by curve (1). This in turn assures that ionization proceeds via the combined effect of collisional and multiphoton ionization processes. Electron diffusion is found to be affecting since the early stages of the laser pulse. At the high pressure, however, the electron density showed a fast increase up to the peak of pulse then it decreases gradually up to its end as shown by curve (2). This decrease is attributed to the role played by the recombination process which acts only during the second half of the pulse (where a high density of free electron can be present).

To confirm these results, calculations are carried out to obtain the temporal variation of the ionization rate at the two values of the gas pressure. These are shown in Figure (4). The deduction which is derived from this figure showed that diffusion process reduces the ionization rate at the low-pressure value shown by curve (1). While at the atmospheric pressure (curve 2 ), this rate is found to be affected mainly by the recombination process during the descending phase of the laser pulse.

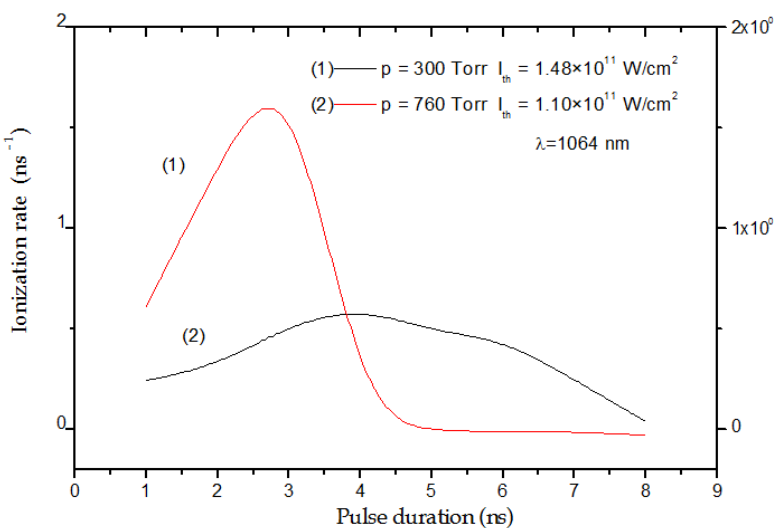

Figure (4): Variation of the number of ionized molecules in the focal volume per nanosecond calculated at the selected values of gas pressure

In this study, it is shown that when multi-photon absorption is the process responsible for gas ionization, the ejected electrons can carry a different amount of energies. The reason for this energy difference is that the energy carried by the ejected electrons depends on the energy of the excited atom from which this electron is ejected (the energy of the absorbed photons required to ionize an excited atom) and the threshold intensity corresponding to the considered gas pressure. It is found that the generated electron from each excited atom carries a different amount of energy $(0.43 \mathrm{eV}$ and $0.86 \mathrm{eV}$ ) for the lower and upper excited states, respectively. These energies match the energy range corresponding to the Ramseur minimum for the momentum transfer cross section, given by Ramseur. [15] This in turn clarifies the important role played by the electron energy gain during the early stages of the breakdown phenomenon. These electrons can be lost by diffusion which in turn decreases the rate of energy gain from the electric field associated with the laser radiation.

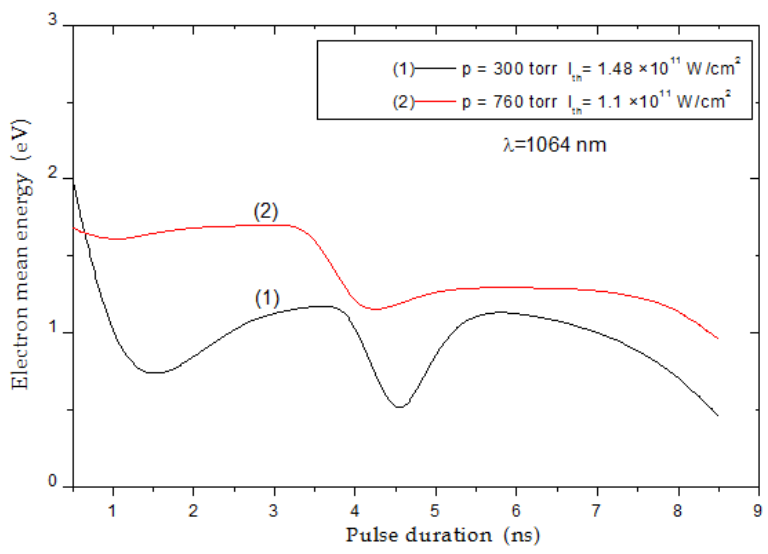

Figure (5) Variation of electron mean energy as a function of time calculated for the two pressure values 
Therefore, it was necessary to study the temporal variation of the electron mean energy. Figure (5) shows this relation for the two selected pressure values. Curve (1) represents the low-pressure region while curve (2) signifies the atmospheric regime. From this figure, in general, the electrons mean energy increases as the gas pressure increases as shown by curves (1) and (2). This increase was attributed to the effect of the loss of electron energy through inelastic collisional processes along with electron loss by recombination which is found to be more effective as the gas pressure increases. It is noticed here that at the low-pressure region the electron mean energy undergoes a drop during the ascending phase of the pulse. This could be attributed to the energy loss through atomic excitation followed by photo-ionization. The minima appeared in the descending phase of the laser pulse refers to the diffusion of energetic electrons from the focal volume. On the contrary, curve (2) showed an almost constant value during the first part of the laser pulse at $1.7 \mathrm{eV}$ followed by a decrease reaching a value of $\sim 1.14 \mathrm{eV}$ which continues up to the end of the pulse. This result could be explained by the fact that electrons first accumulate enough energy to undergo inelastic collisions leading to excitation and ionization of ground state atoms. This, in turn, leads to a high

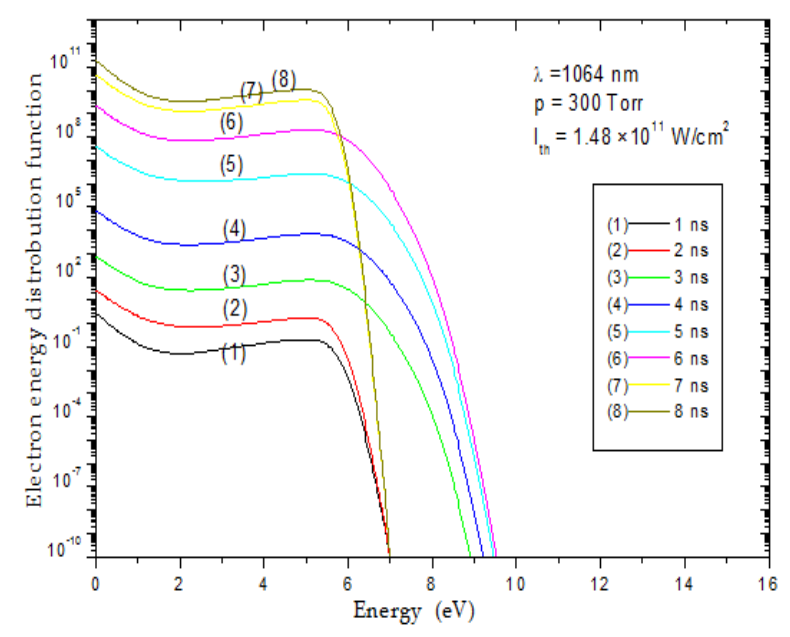

population of the free electrons and positive ions, hence increases the rate of the recombination processes.

To get a deeper inspection of the correlation between the above mentioned physical processes and gas pressure, calculations are carried out to obtain the variation of the EEDF during the laser pulse at the two pressure values. These are shown in Figure (6). It is found that at the low pressure the shape of the EEDF does not vary with the time. The rate of increase of its value follows the pulse shape (i.e. it increases gradually then it grows faster up to the peak. Beyond the peak, it shows symmetric growth which becomes slower as the pulse goes towards its end. It is noticed here that the tail of the EEDF calculated at the end of the pulse is directed towards the low energy region. This behavior explains the effect of electron loss by diffusion. At the high pressure value, however, the EEDF is found to suffer a fast increase during the first half of the pulse. After the peak, its value showed almost coincidence up to its end where the tail is also directed towards the low energy region. This behavior is consistent with the temporal variation of the electrons density shown in Figure(3).

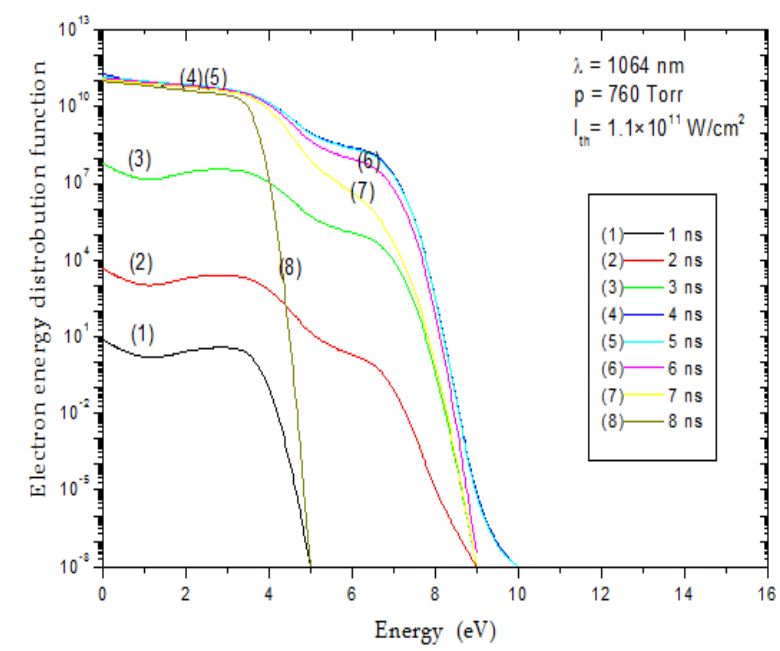

Figure (6): The temporal variation of the EEDF for the considered gas pressures 300 torr (left-hand side) and 760 torr (right-hand side)

This study clarifies that at the low gas pressure value, the breakdown of argon is controlled by the collisional ionization of the excited states in addition to the minor contribution of multiphoton ionization process. At high-pressure value this phenomenon is mainly governed by collisional processes. The shift of the EEDF tail towards low energy region reflects the effect of loss processes. Accordingly, it is necessary to study of the real 
contribution of the loss processes to the electron growth rate.

\section{Effect of loss processes}

To determine the loss processes against gas pressure, calculations are performed to obtain the threshold intensity under the sole action of either diffusion losses or electron recombination. These are shown in Figure(7) where curve (1) represents the absence of diffusion while curve (2) illustrates the case of ignoring recombination. For comparison, the experimentally measured thresholds are shown on this figure as scattered solid circles. It is clear from curve (1) that omission of electron diffusion results in a pronounced deviation between the calculated threshold and the measured ones over the lowpressure regime (25 torr- 300 torr). On the other hand, ignoring recombination losses leads to a clear divergence between the calculated threshold intensity and the measured ones over the high pressure region (300 torr- 760 torr). From this figure, it is shown that diffusion losses are acting over the low-pressure region while recombination has their effect on the high-pressure regime.

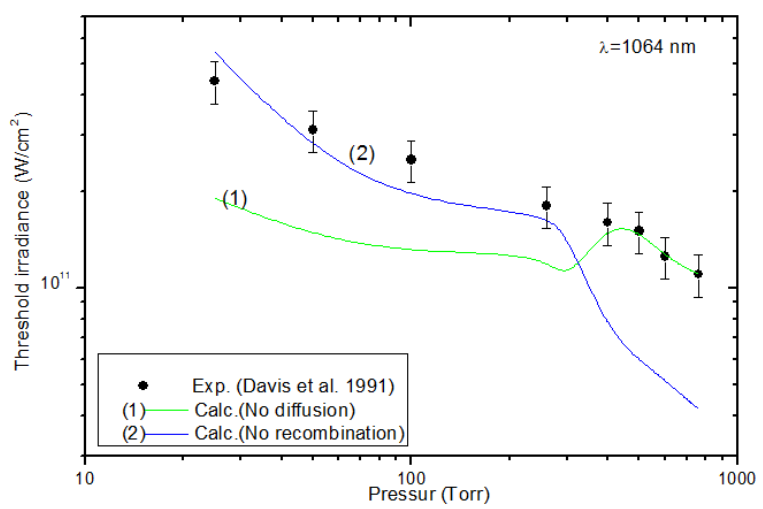

Figure (7): The calculated threshold intensity in the absence of; diffusion (curve 1) and recombination (curve 2) in comparison with the measured experimental ones given by Davis et al. (1991) [1]

Therefore, to identify the exact role of each loss process, curve (1) in Figure (8) demonstrates the ratio of the measured thresholds to the calculated ones in the absence of diffusion while curve (2) represent this ratio when recombination is omitted. From this figure, it is clear that the values of this ratio start with high value $\sim 2.8$ over the lowpressure region followed by a noticeable decrease reaching a constant value of exactly one. This means that diffusion effect is prominent only over the range 25 torr -400 torr. On the contrary, curve
(2) showed a reverse case where the ratio of the threshold intensity started with a constant value of $\sim 1$ then an increase is observed which continues up to the atmospheric pressure with a value $\sim 2.6$.

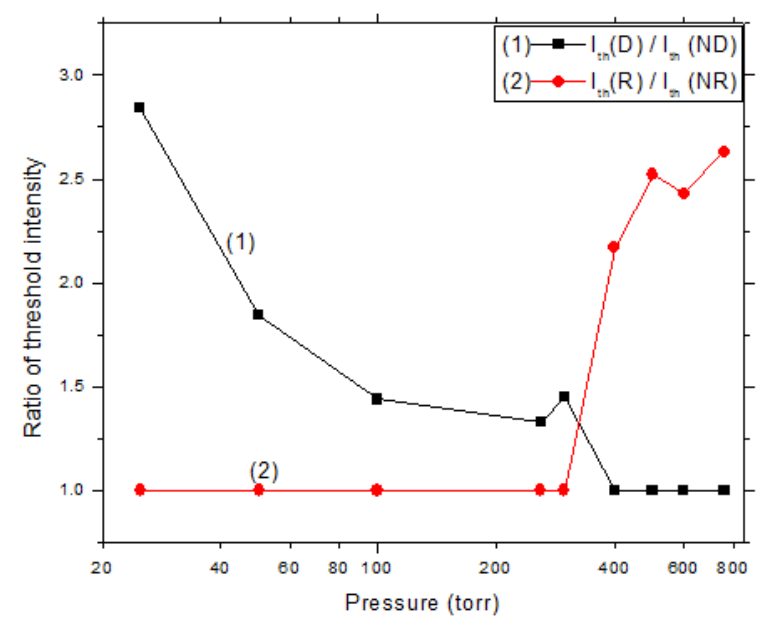

Figure (8): The ratio of measured to calculated threshold intensities for; the absence of diffusion, curve (1), and omission of recombination, curve (2)

This result reveals that at pressures below 300 torr, diffusion loss is the only loss process that competes for the electron generation mechanisms. Also, electron recombination could be the main source of electron losses which determine the threshold intensity and hence plasma ignition at pressures exceeding 300 torr.

\section{Conclusion}

This study is devoted to investigating the threshold intensity dependence on the gas breakdown for argon irradiated with IR laser source at wavelength $1064 \mathrm{~nm}$ and pulse width of $8.5 \mathrm{~ns}$. The investigation aimed to identify the physical mechanisms which are responsible for the determination of the threshold intensity against gas pressure. The modified model is applied to obtain the threshold intensity as a function of gas pressure taken into account all the various physical mechanisms that might take place during thin the interaction region. A comparison between the calculated and measured thresholds showed fair agreement which validates the model. Calculations of the EEDF and its parameters clarified the exact correlation between gas pressure and the physical mechanisms involved in the breakdown phenomenon leading to the generation and depletion of the electron density. Studying the sole action of the diffusion and recombination processes has proven the significant contribution 
of these loss processes in relation to the gas pressure in the breakdown of argon corresponding to the experimental condition under investigation. This result showed that ionization depends mainly on inelastic collisional processes. These processes might be slowed down due to the pronounced Ramseur minima observed in the variation of the momentum transfer cross section with the electron energy for this gas. This in turn could explain the high thresholds observed in this experiment for the IR laser wavelength.

\section{References}

1- J.P. Davis, A.L. Smith, C. Giranda and M. Squicciarini, Appl. Optics; 30, 4358 (1991).

2- C.J. Evans and Y.E.E-D. Gamal, J. Phys. D: Appl. Phys.; 16, 1901 (1980).

3- Y.E.E-D Gamal and M.S. Shafik, J. Phys. D: Appl. Phys.; 26, 767 (1993).

4- J.B. Simeonsson and A.W. Miziolek, Appl. Phys. B; 59, 1 (1994).

5- R. Tambay and R.K. Thareja, J. Appl. Phys.; 70 (5), 2890 (1996).

6- I.C.E. Turcu, M.C. Gower and P. Huntington, Opt. Commun.; 134, 66 (1997).

7- X.Phuoc, Optics Communication; 175, 419 (2000).

8- T. Endo, Laser Ignition Conference (LIC), Optical Society of America, OCIS codes: 140.3440 (2015).

9- Y.E.E.-D. Gamal, M.A.H. Mahmoud and N.D.A. Dawood, Eur. Phys. J.D.; 68, 206 DOI: 10.1140/epjd/e2014-50111-x (2014).

10- Y.E.E.-D. Gamal and G. Abdellatif, Appl. Phys. B.; 00340, 1 DOI 10.1007/s00340-014-5807-0 (2014).

11- ) K.A. Hamam, K.A. Elsayed and Y.E.E. Gamal, Appl. Phys. B.; 123, 89 DOI 10.1007/s00340-0176652-8 (2017).

12- M. Hayatshi, Reo. Atomic Data IPPJ-AM-19. IPPi Nagoya University, Japan (1981).

13- Y.E Gamal and I.M. Azzouz, J. Phys. D: Appl. Phys., 20, 187 (1987).

14- G. Weyl and D. Rosen, Phys. Rev. A 31, 2300 (1985).

15- C. Ramseur, Ann. Phys. 66, 546 (1921). 